\title{
Investigation on Time Domain Signal to Appropriate Wavelet Transform Threshold Function Selection for Partial Discharge Detection
}

\author{
C. K. Vibhakar and S. A. Kanitkar
}

M. S. University, Vadodara, Gujarat, India

chiragkvi@gmail.com

\begin{abstract}
Dielectric insulation is mostly affected due to Partial discharge (PD) pulses, which are resultants of various stress parameters. Normal acquired signal from any insulating material has two components namely actual partial discharge (PD) pulses and noise. Noise suppression is crucial before any (PD) data analysis. PD pulses are short transient, randomly occurred and non-stationary signals. Recent research shows that the Wavelet transform (WT) has achieved good effect in noise rejection with acquired signal. WT proves its suitability by providing information in time and frequency domains simultaneously. Hence, WT can detect these pulses efficiently. PD signals extraction using WT requires set up of various parameters like, optimal mother wavelet selection, decomposition level selection, threshold rule and function determination. This paper presents the vital issues of WT threshold function determination. Here, a method is envisaged for appropriate WT threshold function selection based on acquired signal analysis. This work is carried out on signal measured from transformer. Also, analysis is carried out on the acquired signal and its WT generated signal. Finally, a comparison is carried out to select optimum threshold function selection for WT signal analysis.
\end{abstract}

Keywords: Partial Discharge, Wavelet transform, Hard threshold function, Soft threshold function, Signal Analysis

\section{Introduction}

Electrical insulation and its life depend on various stress parameters like voltage, temperature, pressure etc. These parameters affect severely on its performance during life span [1]. Insulator failure is mostly due to the breakdown resulted from PD occurrences. Partial discharge (PD) is one of the symptoms of electrical insulation deterioration in high voltage equipment (transformers, bushings, cables, rotating machines etc.). The consequence of gradual and cumulative PD occurrences affects the insulation life over the years. Detection and diagnosis of PD of the inner insulating systems in HV equipments have been widely adopted as a means for insulation condition monitoring.

In actual working phase of any electrical equipment, the harsh environment and significant back-ground electrical noise can swamp the PD signals which are in the range of mill-volt to micro-volt. Noise has been a major challenge to PD on-line measurement. PD signals have a transient, irregular and non-periodical nature. Therefore, statistical characterization or WT method can be powerful technique for analyzing these signals. There is lots of research done on statistical characterization method for PD [2,3]. Due to particularly designed to analyze transient, irregular and non-periodical signals in a time-scale domain, Wavelet transform (WT) can potentially be used to PD signals extraction from severe noise $[4,5]$. Although publications have been made in utilizing the discrete Wavelet transform (DWT) [6] and Wavelet packet transform (WPT) [7] for exacting PD signals. Some important issues for on-line PD measurement are resolved in [4].

Received: October $13^{\text {rd }}, 2010$. Accepted: March $8^{\text {th }}, 2011$ 
During testing it is observed that PD intensity increase/decrease gradually with different stress parameters. In this paper, a method is envisaged for optimum WT threshold function selection from real-time acquired data on Transformer. Also, the newly developed method uses previous data and can be used for anticipating appropriate WT threshold function selection for fore-coming data.

Also, it is necessary to define all WT parameters during hardware realization. This method/work eliminates need of WT threshold function selection requirement which is one of the desired steps.

\section{Wavelet Transform and Signal De-Noising}

Wavelet analysis is an extension of Short-Time Fourier Transform. It allows variable-sized window and produces a time-scale view of the signal (instead of time-frequency). In essence, the technique decomposes a signal into shifted and scaled versions of an original (or mother) wavelet. It is capable of providing the time and frequency information simultaneously. There is requirement of four parameters for WT which are (1) optimal mother wavelet selection; (2) optimal decomposition level selection; (3) threshold rule determination and (4) thresholding function determination.

WT is done through shifting and scaling mother wavelet. Shifting a wavelet means delaying or hastening its onset. Scaling of a wavelet means stretching or compressing it. There is a correspondence between scaling and frequency. Low scale produces a compressed wavelet suitable for rapidly changing details and thus corresponds to high frequency. Similarly, high scale gives a stretched wavelet which characterizes slowly changing features and thus corresponds to low frequency.

Wavelet decomposition removes high-frequency noise from the signal by successive approximations and it provides a simple method to de-noise the signal. Signal decomposition can be achieved by various thresholding methods. It includes threshold value determination and threshold function selection.

The threshold value determines a level which is used for threshold function section. There are four common methods used for threshold value determination which are Universal threshold rule, Minimax threshold rule, Stein unbiased risk threshold rule and Level-dependent threshold rule. Universal threshold rule is used for present analysis which, determines threshold as per equation-1

$$
\lambda_{\text {universal }}=\sigma \sqrt{2 \log (n)}
$$

Where, $\mathrm{n}$ is the total number of data points and $\sigma$ is the standard deviation of noise level.

There are two threshold functions available namely Hard and Soft thresholding [8]. Hard thresholding processes data in such a way that those wavelets coefficients whose absolute values are greater than the threshold are retained while those less than the threshold are set to zero. Derived hard threshold function value $\delta_{\lambda}^{H}$ is shown in equation-2.

$$
\delta_{\lambda}^{H}(x)= \begin{cases}x, & \text { if }|x|>\lambda \\ 0, & \text { otherwise }\end{cases}
$$

Here, $\mathrm{x}$ is input value and $\lambda$ shows threshold value. In soft thresholding the wavelet coefficients below the threshold give zero and the coefficients greater than threshold are

retained and then shrunk toward zero. Derived soft threshold function value $\delta_{\lambda}^{S}$ is shown in equation-3. Hard and soft threshold values are as shown in Figure 1. 


$$
\delta_{\lambda}^{S}(x)= \begin{cases}x-\lambda, & \text { if } x>\lambda \\ 0, & \text { if }|x| \leq \lambda \\ x+\lambda, & \text { if } x<-\lambda\end{cases}
$$

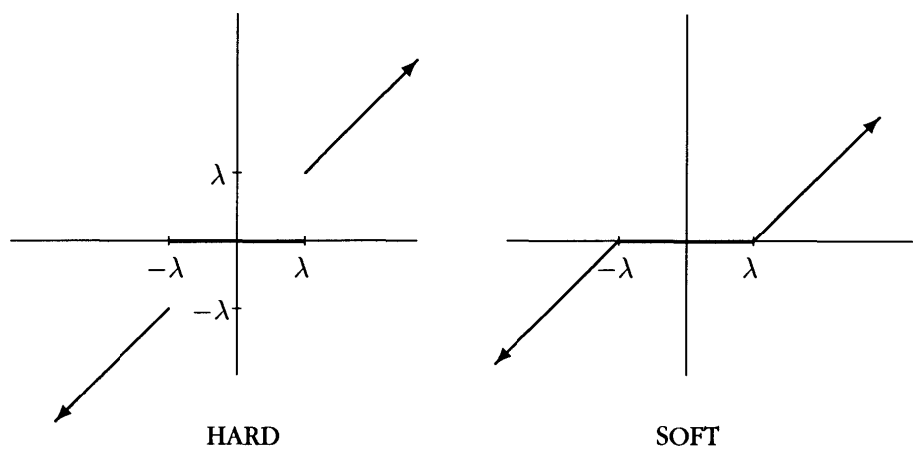

Figure 1. The Hard and Soft threshold function

\section{PD Data Acquisition for Power Transformer}

The experiment is carried out as per Figure 2. It shows, the work is carried out for two phases (1) Measurement and (2) signal acquisition and analysis.

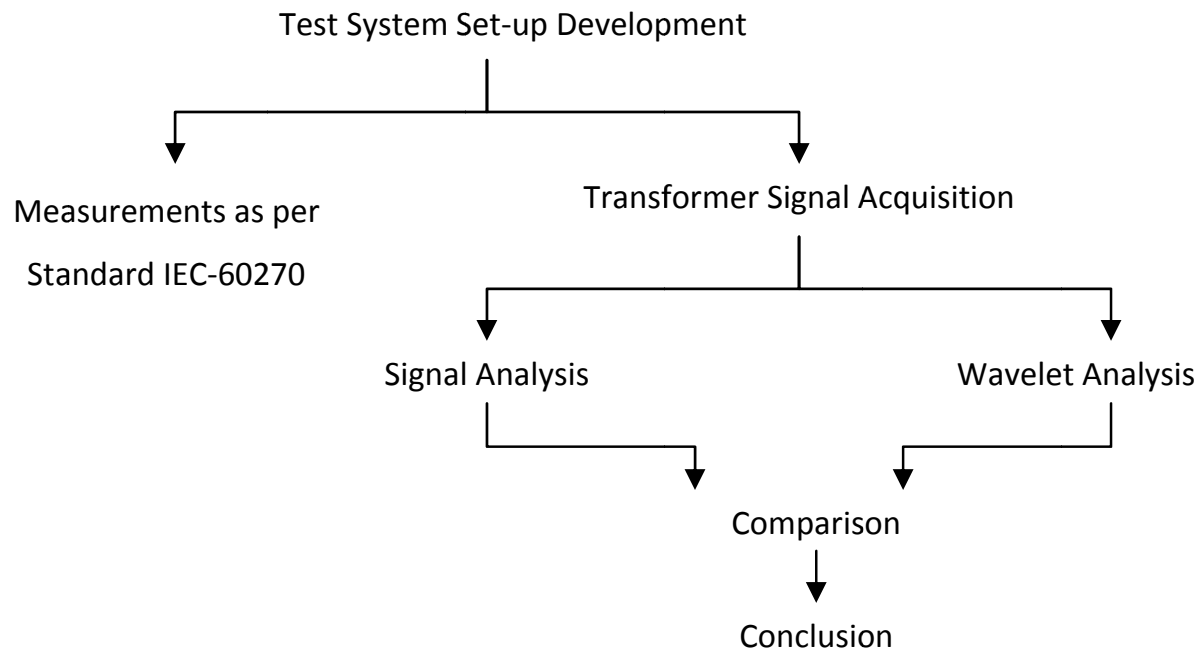

Figure 2. Flow of the experiment and analysis

As shown in the Figure 2, first step is carried out to test set-up development. The set-up is done for 100 MVA, 220/132 kV transformer [9] as per Mtronics made Advanced Partial Discharge Measuring System MPD540. During testing measurements are carried out as per standard IEC-60270. Apart from this, during testing some waveforms are recorded for signal analysis to establish relation between acquired signal and WT. Block diagram of on line PD measurement is shown in Figure 3.

The PD measurements are carried out using Mtronics made Advanced Partial Discharge Measuring System MPD540. This unit comprises data acquisition unit (MPD540) and fiber optics controller (USB502). 
Former consists of a coupling unit (MPD540CPL) and data acquisition unit which is USB based data transfer unit. MPD540CPL is connected high voltage coupling capacitor through unit CPL542 which is also referred to as "quadripole".

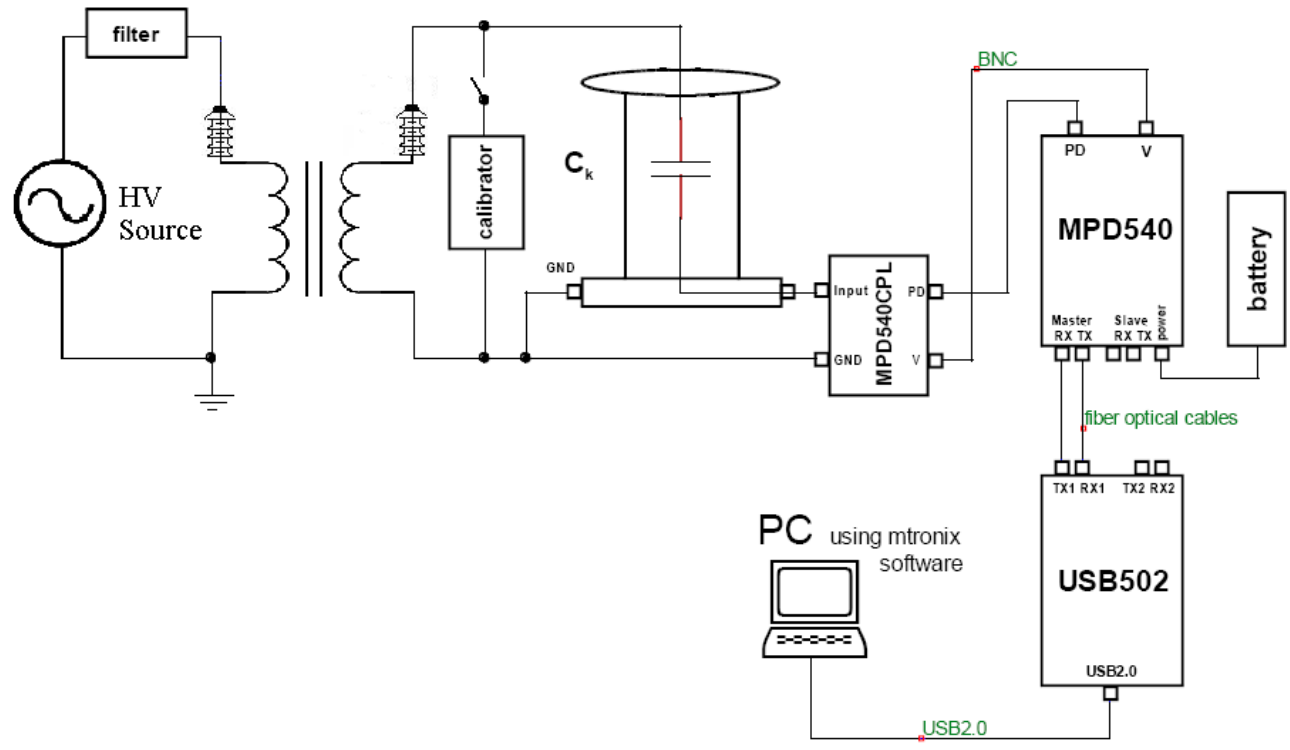

Figure 3. Block diagram of on line PD measurement setup

Before starting the measurements the set-up is calibrated for $500 \mathrm{pC}$ and it meets calibration criteria. PD test bandwidth is kept $300 \mathrm{kHz}$ with PD test maximum voltage $245 \mathrm{kV}$ as per IS standard. The experiment is carried out as per method. Measurements are taken at different voltages and durations as per standard. PD pulse and measurement value is obtained at different range of voltages with respect to time schedule on $\mathrm{HV}$ and $\mathrm{LV}$ phases of transformer. Measurements of PD and test voltages are recorded in measured in Table-1 [10]. As shown in this table, numbers of PD samples are recorded at defined voltage level and time duration. Each set of data are recorded with software which is exported for WT analysis. One of the recorded waveform is shown in Figure 4.

Table 1. Measured PD at different timing conditions

\begin{tabular}{|c|c|c|c|c|c|c|c|c|c|c|c|}
\hline \multirow{2}{*}{$\begin{array}{c}\text { Test } \\
\text { Voltage(kV) }\end{array}$} & \multirow{2}{*}{$\begin{array}{c}\text { Time } \\
\text { Duration }\end{array}$} & $\mathbf{1}$ & $\mathbf{2}$ & $\mathbf{3}$ & $\mathbf{4}$ & $\mathbf{5}$ & $\mathbf{6}$ & $\mathbf{7}$ & $\mathbf{8}$ & $\mathbf{9}$ & $\mathbf{1 0}$ \\
\hline 212.18 & 1 minute & 260 & 280 & 290 & 280 & 270 & - & - & - & - & - \\
\hline 245 & 5 second & 505 & - & - & - & - & - & - & - & - & - \\
\hline 212.18 & 3 minute & 450 & 500 & 600 & 1000 & 1500 & 2480 & 3484 & 5480 & 6000 & 6282 \\
\hline
\end{tabular}

In second part (Transformer signal acquisition and analysis as per Figure 2) the measured waveforms are stored in PC (at different time instance). The Mtronics software has data export feature which converts recorded signal values to time stamped signal samples. Also, the extracted samples are stored in text file and further analysis is carried out.

This text file data (reference data) is given to developed program and further WT analysis was carried out to get PD pulses. WT has removed noise samples and estimated PD pulses. 


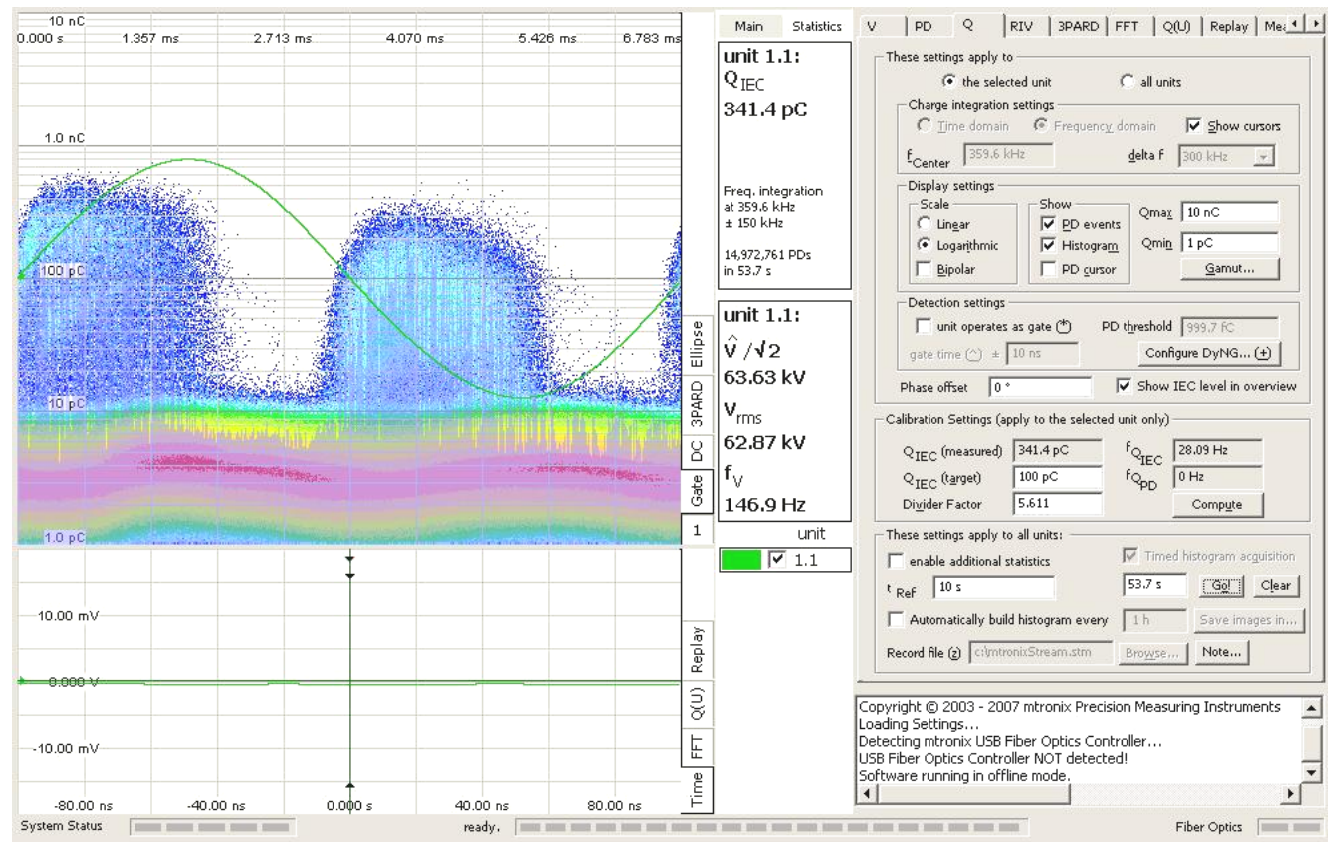

Figure 4. Snapshot of one of the recorded waveform through Mtronics Software

\section{Acquired Signal Analysis In Time Domain and WT Domain}

During the experiment several data sets were recorded for analysis. These recorded data contains PD signals with noise. Each recorded data set is transferred to compatible data analysis software where two types of analyses are carried out on signal (Figure 2). First, analysis is carried out on acquired signal and another on the WT analysis [11, 12]. Then, both analyses are compared to derive a conclusion.

Figure 5 shows block diagram of time domain signal. Partial discharge signals are bidirectional in nature. Also, standard deviation and other statistical methods can not be directly useful for signal analysis. So, the direct analysis is not viable for this signal and it is to be separated between positive and negative samples. Further analysis is done separately on positive and negative samples and finally both are separately compared for result derivation.

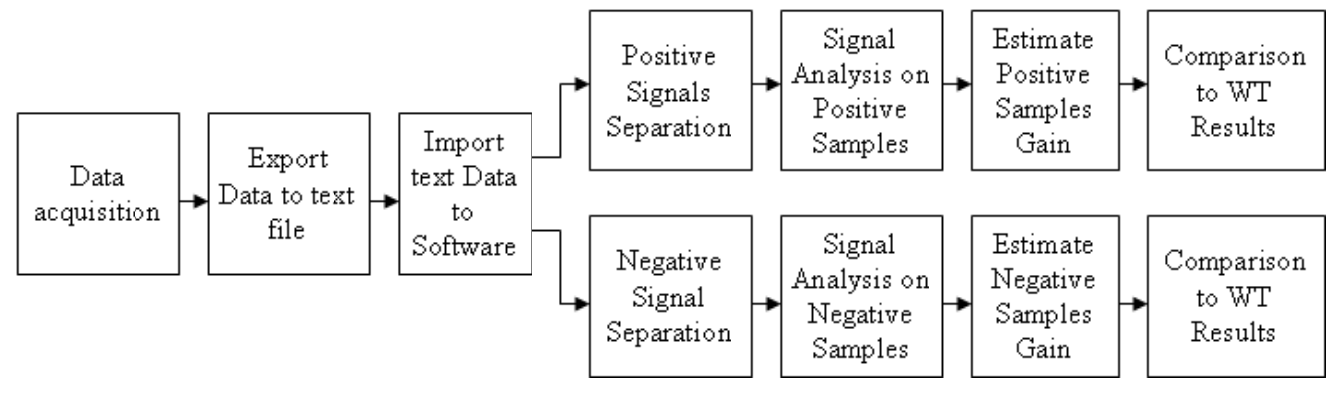

Figure 5. Block Diagram for acquired signal analysis

Let us say, $S n$ is a signal having PD pulses. The sampled signals can be expressed as in (4).

$$
S n=S n_{\text {pos }}+S n_{\text {neg }}
$$


Where $S n_{\text {pos }}$ is total positive samples and $S n_{\text {neg }}$ is total negative samples. A developed program separates positive and negative samples and their average is found out as stated by (5) and (6).

$$
\begin{aligned}
& A v g_{\text {pos }}=\left(\Sigma S n_{\text {pos }}\right) / N_{\text {pos }} \\
& A_{\text {vg }}=\left(\Sigma S n_{\text {neg }}\right) / N_{\text {neg }}
\end{aligned}
$$
where, $A v g_{\text {pos }}$ is positive samples average, $A v g_{\text {neg }}$ is negative samples average, $N_{\text {pos }}$ is number of positive samples and $N_{\text {neg }}$ is number of negative samples. PD pulses estimation is carried out as per (7) and (8).

$$
\begin{aligned}
& A v g_{\text {est_pos_PD }}=\Sigma\left(S n_{\text {pos }}-A v g_{\text {pos }}\right) / N_{\text {est_pos_PD }}, \quad \text { if } S n_{\text {pos }}>\left(p^{*} A v g_{\text {pos }}\right) \\
& =0, \quad \text { if } S n_{\text {pos }} \leq\left(p^{*} A v g_{\text {pos }}\right) \\
& A v g_{\text {est_neg_PD }} \quad=\Sigma\left(S n_{\text {neg }}-A v g_{\text {neg }}\right) / N_{\text {est_neg_PD }}, \quad \text { if } S n_{\text {neg }}<\left(p^{*} A v g_{\text {neg }}\right) \\
& =0 \text {, } \\
& \text { if } S n_{\text {neg }} \geq\left(p^{*} A v g_{\text {neg }}\right)
\end{aligned}
$$

where, $A v g_{\text {est_pos_PD }}$ is average of pulses which are above $A v g_{\text {pos }}, N_{\text {est_pos_PD }}$ is number of pulses whose value is above $p^{*} A v g_{\text {pos }}, A v g_{\text {est_neg_PD }}$ is average of pulses which are below $A v g_{\text {neg }}$, $N_{\text {est_neg_PD }}$ is number of pulses whose value is below $p^{*} A v g_{\text {neg }}$ and $p$ is the number which is used to estimate PD pulse amplitude. This experiment is carried out for different values of $p$ which are 1, 1.5, 2 and 3. However, for $p=2$ best result is achieved among all 13 data set files (each data set has more then 5000 samples).

A positive pulse ratio of estimated positive pulses and average positive signal samples determines positive PD pulse intensity and vice versa. These ratios are indicated in (9) and (10).

$$
\begin{aligned}
& R_{\text {pos }}=A v g_{\text {est } \_ \text {pos_PD }} / A v g_{\text {pos }} \\
& R_{\text {neg }}=A v g_{\text {est_neg_PD }} / A v g_{\text {neg }}
\end{aligned}
$$

where $R_{\text {pos }}$ and $R_{\text {neg }}$ are ratios for positive and negative estimated PD pulses respectively. This ratio estimates PD pulses intensity on actual signal. $R_{\text {pos }}$ high indicates that the signal has low noise contribution and high PD amplitude and vice versa. During the experiment $R_{\text {pos }}$ and $R_{\text {neg }}$ ratios are found normally equal. These ratios are used for comparison with WT determined signals.

Figure 6 shows block diagram for WT analysis for determine Hard threshold function gain over soft threshold function. As discussed earlier, universal threshold method decides threshold value for present analyses.

WT is applied on signal with universal threshold value for hard and soft threshold function. Hard threshold function retains original values. So, it always gives better performance over soft threshold. This performance is referred as gain $\left(R_{p o s}\right.$ and $\left.R_{n e g}\right)$ and it is used for comparison with original signal. During practical experiment, 13 different data sets are stored separately at different time interval. Both analyses are carried out on each of the data set.

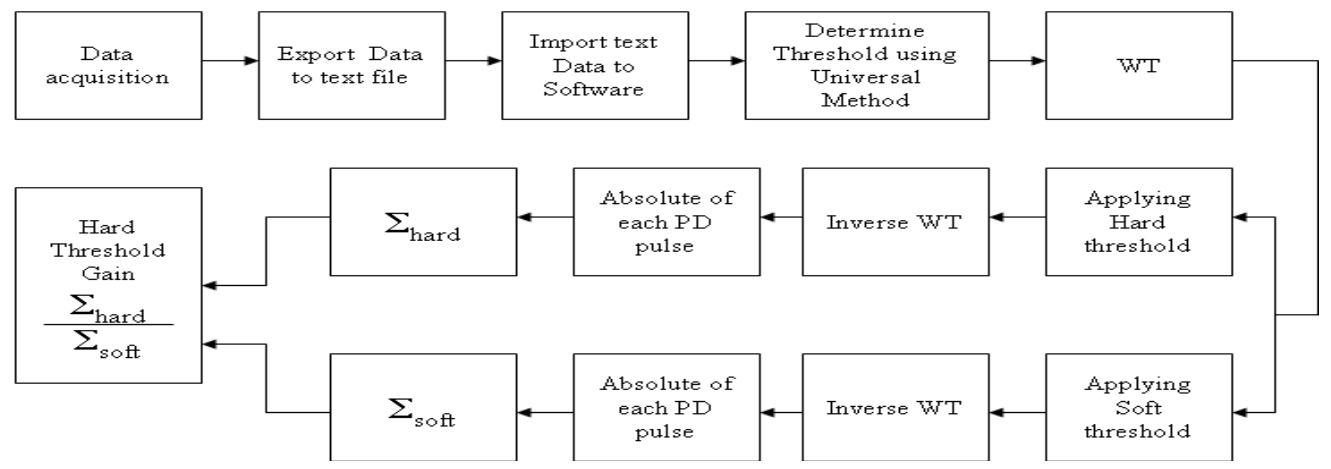

Figure 6 Block Diagram for WT for determine Hard Threshold Function Gain 
Two sets of WT results of with hard and soft threshold function are shown in Figure 7 and Figure 8.

Figure 7 shows the data set where PD values are much higher then noise. Here, $R_{\text {pos and }} R_{\text {neg }}$ are much higher. In this case, the PD signal derived using hard and soft threshold are nearly same.
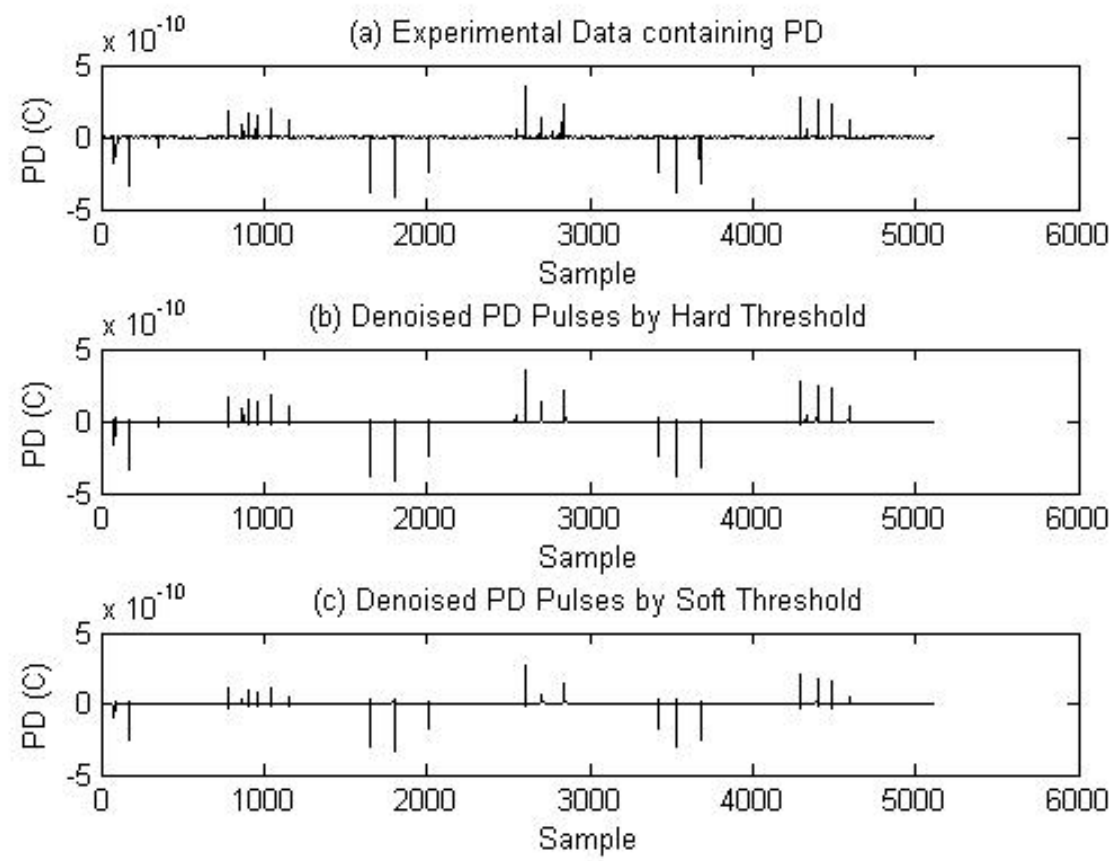

Figure 7 Effect of hard and soft threshold selection on experimental data set-1 (refer Figure 9).

Figure 8 also shows the data set where PD values are small. Here, $R_{\text {pos and }} R_{\text {neg }}$ are much less. In this case, threshold function selection plays important role and only hard threshold function can detect PD signals.
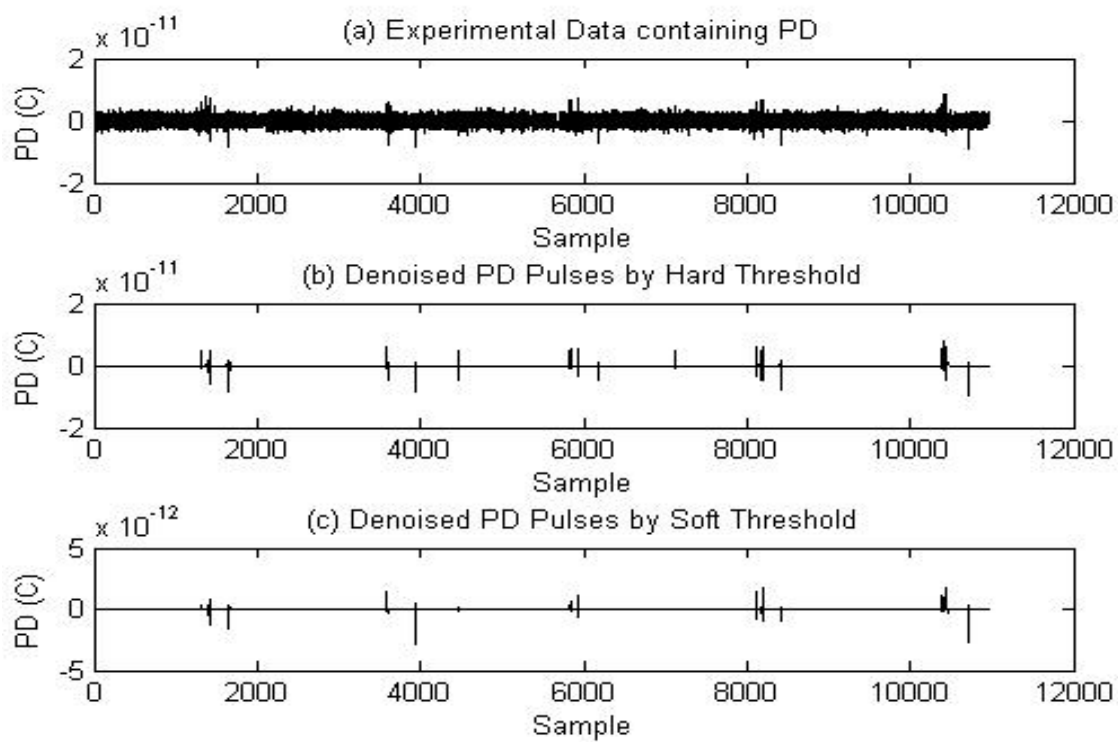
Figure 8 Effect of hard and soft threshold selection on experimental data set-7 (refer Figure 9)

Finally, a comparison is formed on each data set and it is shown in Figure 9. It shows all 13 stored data sets analysis and results. Figure 7 and Figure 8 are detail analysis of record set- 1 and record set-7 respectively.

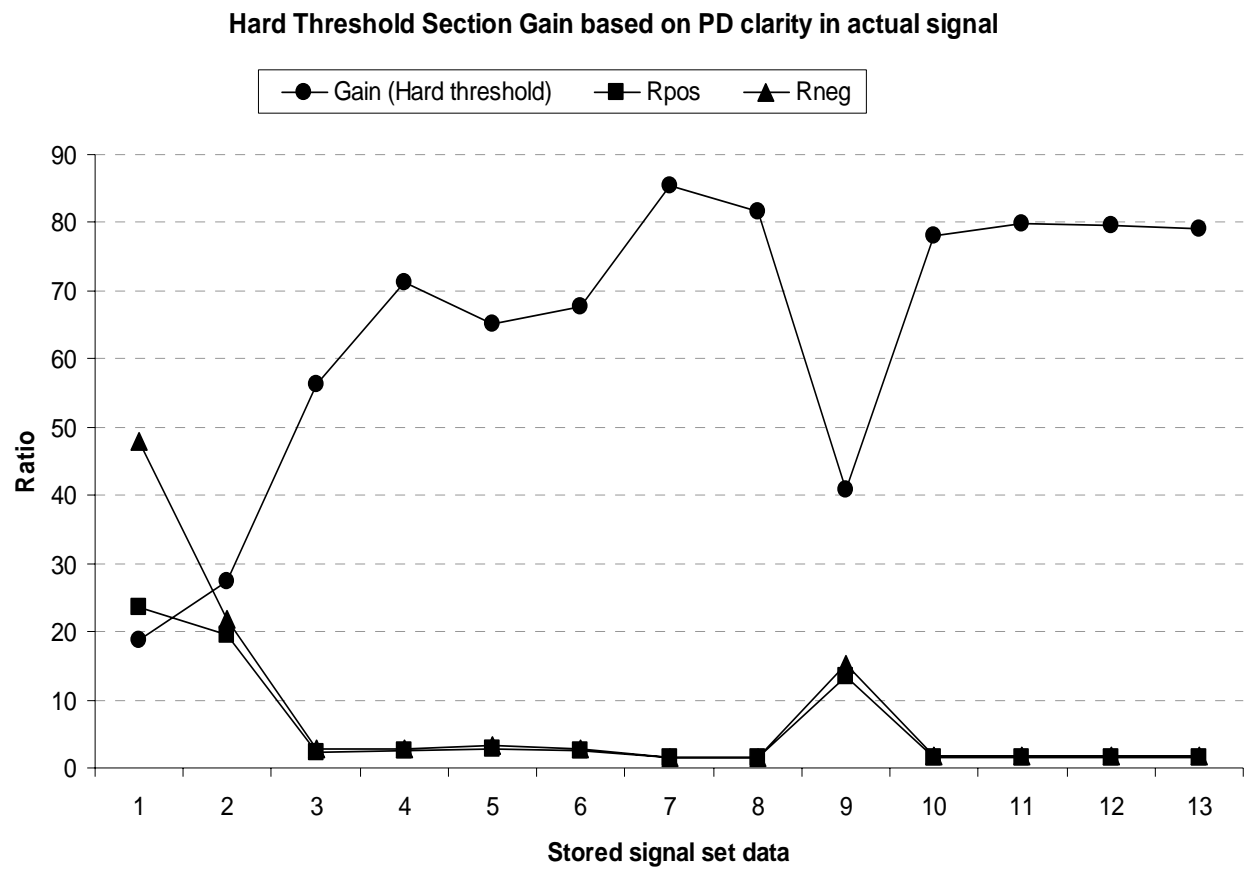

Figure 9 Comparison of Hard threshold gain on actual signal (for $p=2$ )

As shown in Figure 9, when $R_{\text {pos }}$ and $R_{\text {neg }}$ are higher then hard threshold gain is less and vice versa. In other words, when acquired signal have unclear PD pulses or more noise contribution; then only hard threshold gives good results whereas in other conditions both threshold functions gives near same results.

\section{Conclusion}

In this paper, simulated PD signals were extracted with Wavelet transform. A PD pulse denoising is carried out using universal threshold with hard and soft threshold function. Also on actual signal, analysis is carried to determine in advance which threshold function is to be selected. Based on actual data and analysis, it is concluded that if the signal has low PD pulse intensity then there is great advantage of hard threshold function, but when noise contribution is low compared to PD pulses then there is marginal advantage of hard threshold function over soft threshold function (Figure 9 stored data sets 1, 2 and 9). So, based on actual data expectation early threshold function can be estimated.

Further, a hardware can be designed which, chooses appropriate threshold function for optimum WT threshold function selection based on present time domain data stream.

\section{References}

[1] C. K. Vibhakar and S. A. Kanitkar "Investigation and modeling of epoxies aging tests under the effect of variation of parameters on partial discharge", IEEE conference-Trans. ISBN:978-1-4244-4331-4.

[2] B. T. Phung, “Computer-based Partial Discharge Detection and Characterization”, Ph. D thesis, University of NSW, Australia, 1997. 
[3] R. E. James and B. T. Phung, "Development of Computer-Based Measurement Systems for Recording and Analysis of Partial Discharge Patterns", IEEE-DEI Trans., Vol.2, No.5, Oct. 1995, pp. 838-856

[4] Evagorou, D.; Kyprianou, A.; Lewin, P.L.; Stavrou, A.; Efthymiou, V.; Georghiou, G.E.; "Evaluation of Partial Discharge Denoising using the Wavelet Packets Transform as a Preprocessing Step for Classification”, IEEE Electrical Insulation and Dielectric Phenomena, 2008. CEIDP 2008. Annual Report Conference on; 26-29 Oct. 2008; pp 387390.

[5] Yamada Ioya, Inui Akifumi, Kawaguchi Yoshihiro; "Distinction of Partial Discharge Signal from Noisy Signal by Daubechies Wavelet Transform"; Transactions of the Institute of Electrical Engineers of Japan. B; Vol.123; No.8; 2003; pp. 988-994.

[6] X. Zhou, C. Zhou and I. J. Kemp, "An Improved Methodology for Application of Wavelet Transform to Partial Discharge Measurement Denoising,", IEEE Transaction on Electrical Insulation and Dielectrics, Vol. 12, No.3, pp.586-594, June, 2005.

[7] C. S. Chang, J. Jin and S. Kumar, "Denoising of Partial Discharge Signals in Wavelet Packets Domain,” IEE Proc.-Sci. Meas. Technol, Vol.152, No.3, 2005, pp.129-139

[8] F. Abramovich, F. Sapatinas, and B. W. Silverman ,"Wavelet thresholding via a Bayesian approach,” J. R. Stat. Soc., B 60: 725-749, (1998).

[9] S. N. Hettiwatte, P. A. Crossley, Z. D. Wang, , A. Darwin and G. Edwards, "Simulation of a Transformer Winding for Partial Discharge Propagation Studies”, IEEE 2002.

[10] H. Okubo, H. Nayakawa, "A novel technique for partial discharge and breakdown investigation based on current pulse waveform analysis", IEEE Trans., on Dielectric and Electrical insulation, Vol.12, pp 736-744, 2005.

[11] R. Todd Odgen, "Essential Wavelets for statistical Applications and Data Analysis", ISBN 0-8176-3864-4.

[12] F. Abramovich , T. C. Bailey, T. Sapatinas, "Wavelet analysis and its statistical application,” The Statistician 49:1-29, 2000

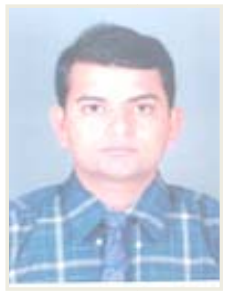

C. K. Vibhakara was born in Jamnagar, Gujarat, India, on March $25^{\text {th }} 1974$. He obtained his B. E. degree from Nagpur University and M. E. Birla Vishvakarma Mahavidyalaya Engg. College, Vallabh Vidhya Nagar, Sardar Patel University. He is presently pursuing the Ph.D. degree at M. S. University, Baroda.

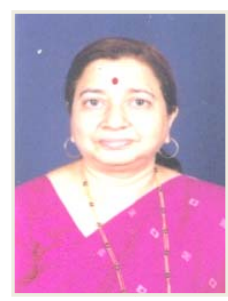

S. A. Kanitkar obtained her B. E. degree from Devi Ahliya university, Indore and M. E. degree from the M.S.University, Vadodara. She is presently Professor and Head of the. Dept. of Electrical Engg., Faculty of Technology, M. S. University, Vadodara. 